\title{
Téoros
}

Revue de recherche en tourisme

\section{Le mythe des retombées économiques}

\section{L'intervention gouvernementale dans le secteur touristique}

\section{Marcel Côté}

Volume 3, numéro 2, juillet 1984

L'intervention étatique dans le domaine du tourisme : premiers

coups de sonde

URI : https://id.erudit.org/iderudit/1080798ar

DOI : https://doi.org/10.7202/1080798ar

Aller au sommaire du numéro

\section{Éditeur(s)}

Université du Québec à Montréal

ISSN

0712-8657 (imprimé)

1923-2705 (numérique)

Découvrir la revue

Citer cet article

Côté, M. (1984). Le mythe des retombées économiques : l'intervention gouvernementale dans le secteur touristique. Téoros, 3(2), 2-6.

https://doi.org/10.7202/1080798ar d'utilisation que vous pouvez consulter en ligne.

https://apropos.erudit.org/fr/usagers/politique-dutilisation/ 


\title{
Le mythe des retombées économiques
}

\author{
par Marcel Côté*
}

Le tourisme est I'objet des sollicitudes croissantes des gouvernements. Cette largesse repose sur deux hypotheses: la croyance que le développement du tourisme a un impact économique important et la très haute visibilité du développement touristique. Nous voudrions dans ce texte faire le tour de la question. En première partie, nous tenterons de situer économiquement le tourisme. Dans une deuxiême partie, nous traiterons de l'impact économique du tourisme. La troisième partie portera sur l'intervention gouvernementale et, en conclusion, nous ferons état de nos vues personnelles sur l'ampleur et l'à-propos de cette présence des gouvernements à titre d'agents économiques dans le secteur touristique.

\section{L'importance économique du tourisme}

Comme activité économique, le tourisme souffre lou bénéficiel d'une définition relativement élastique. II est en effet temporaire. II est dono difficile de cerner l'activité touristique sur le plan économique car il s'agit d'un amalgame d'activités caractérisé par le fait que le consommateur est en situation de déplacement, ce qui crée beaucoup de confusion sur le plan analytique. Voici trois exemples de confusion qui illustrent bien les difficultés méthodologiques.

1. Quand se termine la promenade et quand commence le voyage? Le tourisme est associé au voyage. II est difficile mais très important de préciser quand commence le voyage car il se fait un très grand nombre de déplacements de petites distances. En somme, devient-on touriste aussitot qu'on s'absente 24 heures de chez-soi, ou bien l'est-on lorsqu'on visite la ville voisine pour quelques heures, ou bien, comme aux Etats-Unis, devient-on touriste après avoir franchi 100 milles?

\footnotetext{
*L'auteur est économiste et associé principal de SECOR inc., une entreprise de consultation stratégique de Montréal.
}

2. Où se termine le tourisme et oủ commence le loisir? La frontière entre le tourisme et le loisir est d'autant plus difficile que la pratique de certaines activités de loisir exige des déplacements. Les skieurs de Montréal sont-ils des touristes au MontTremblant? Les chasseurs? Les membres du Club nautique du lac des Deux-Montagnes? Les campeurs dans des roulottes à Ste-Rosalie?

3. Qu'est-ce que le tourisme d'affaires? Tous les voyages d'affaires sontils du tourisme? La propriétaire d'une boutique de mode de Chicoutimi qui fait ses achats d'automne a Montréal, est-elle une touriste d'affaires? Et si elle allait â Vancouver? Une réunion de planification de cadres de I'Alcan au siége social de Montréal constitue-telle du tourisme d'affaires?

L'élasticité d'une définition plaìt bien à ceux qui aiment les grosses industries. Mais en embrassant trop, l'industrie risque de se dénaturer. Elle n'a plus d'homogénéité, ni de début, ni de fin. II devient alors plus difficile de définir des politiques coherrentes, d'organiser en fonction d'objectifs communs, de savoir où l'on va et d'où on est venu. Trop d'éléments disparates sont regroupés artificiellement.

En fait, le problème est lié au fait que le tourisme n'est pas une industrie. Une industrie peut être définie comme un ensemble d'organisations, généralement des entreprises, qui partagent un corpus de technologie, des marchess, et parfois des sources d'approvisionnement. On peut identifier ainsi les pâtes et papiers, les institutions financières, l'agriculture, les ateliers d'usinage, etc.

Le tourisme comprend un ensemble hétérogene d'activités et chevauche donc plusieurs industries. Le tourisme comprend, entre autres, le voyage aerien, l'hôtellerie des grandes villes, les commerces s'adressant aux automobilistes, aux visiteurs de lieux touristiques, etc. La difficulté de définir le tourisme comme industrie peut être mise en evidence par la question suivante: quelle autre uindustrien est aussi multiforme que I'industrie du tourisme? Aucune, si ce n'est l'industrie du loisir, si celle-ci existe. Or comment définit-on l'industrie du loisir? Doit-on compter les dépenses au MontTremblant: la bière bue au forum et au Stade Olympique; la radio et la télévision; les pages de sports dans les journeaux; les budgets des bibliothèques: les voitures sports et un pourcentage des ventes d'aspirine, etc.

C'est un peu ce que l'on fait lorsqu'on estime la taille de l'industrie touristique. Toutes les dépenses encourues lors de déplacements durant plus d'une nuit au Canada ou de plus de 100 milles aux ĖtatsUnis sont classées comme des adépenses touristiquess. On y retrouve donc beaucoup de bière et d'aspirine, de même que des depenses de toutes sortes qui ne sont pas associees à l'industrie du tourisme $(e x$, : achats de bibelots, vêtements, etc.l.

Selon Statistique Canada, les dépenses touristiques des Canadiens équivalent a environ $5 \%$ du produit national brut. ${ }^{\text {(1) }}$ il s'agit de l'estimé des dépenses par les Canadiens lors de séjour de plus d'une nuit hors de chez-eux. En 1981, des dépenses étaient de $\$ 6,5$ milliards, alors que le produit national brut canadien était de $\$ 331$ milliards $^{(2)}$. Ces dépenses touristiques reprósentaient $9 \%$ des dépenses de consommation au Canada. Evidemment. une partie de ces dépenses étaít failte à l'étranger, soit environ $30 \%$. Par contre, les étrangers en visite au Canada ont aussi dépensé significativement, quoiqu un peu moins que les Canadiens à l'étranger. Environ le quart des adépenses de tourismes au Canada sont le lot d'étrangers.

Pour le Québec, on peut présumer que la situation est la mème. $5 \%$ du produit national brut québécois serait dủ à des dépenses touristiques, soit $\$ 5$ milliards au Québec. Les dépenses à l'extérieur du Canada devraient représenter environ $30 \%$ des dépenses des touristes québécois. Les dépenses des Québécois 


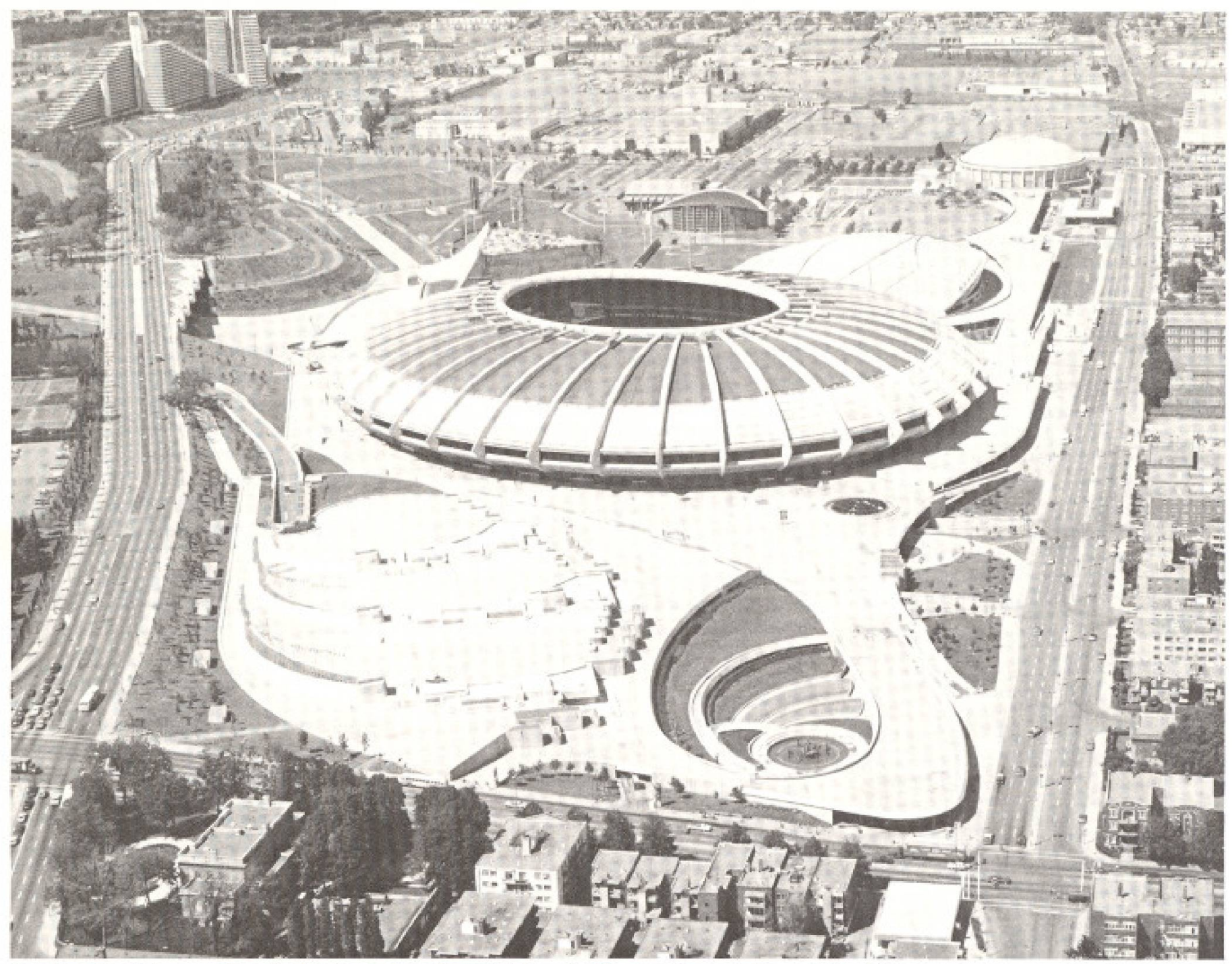

Ce n'est toutefois pas aujourd'hui qu'on arreatera les politiciens de depenser l'argent des contribuables .

En avant les jeux; les gouvernements sont généreux!

qui se déplacent dans les autres provinces pourraient représenter $20 \%$ du total et le reste serait des dépenses faites au Québec par des Québécois.

La contribution des touristes étrangers ả la dépense nationale en biens et services, n'est pas négligeable. Selon l'OCDE, cette part serait de l'ordre de $2,6 \%$ au Canada ${ }^{\mid 3 \%}$. Dans des pays comme la Belgique, le Danemark et la Hollande, elle serait de l'ordre de $4 \%$. Il est fort plausible qu'il en soit ainsi au Québec, en ce qui à trait aux non-Québécois.

Les dépenses faites à l'occasion de voyages aériens representent une partie importante, soit $40 \%$, contre près de $60 \%$ pour les voyages en automobile. De plus, le marché global se divise inégalement entre trois segments : Ie voyage de plaisir (environ $55 \%$ des dépenses totales); les visites à des parents ou amis lenviron $20 \%$ l; et le voyage d'affaires lenviron $25 \%^{(4)}$.

Enfin, la composition des dépenses est aussi importante. Trois secteurs dominent. En premier lieu, le transport, avec environ le quart de la dépense et qui se subdivise principalement entre le transport aérien et les services automobiles. Dans les deux cas, le carburant et l'amortissement de l'équipement sont les principales sources de dépenses. En deuxiême lieu, on retrouve, sensiblement à parts égales, I'hébergement et la restauration. Ceci n'est pas surprenant. En effet, selon les statistiques américaines qui sont assez précises sur le sujet, pour chaque vovage de plus de 100 milles, il y a en moyenne près de 5 nuits d'hébergement et donc une quinzaine de repas ${ }^{(5)}$. II faut bien se nourrir et se coucher et les dépenses à cette fin pesent lourd dans le budget du touriste, immédiatement après ses dépenses de transport. Ensemble, ces trois secteurs comptent entre $70 \%$ et $80 \%$ des dépenses totales par les touristes.

Les autres dépenses sont réparties dans un grand nombre de secteurs dont plusieurs ne sont pas reliés au tourisme. Les postes fiscaux sont aussi importants (taxe d'amusement, taxe de vente, etc-).

La dépense touristique $n^{\prime}$ est donc certes pas â négliger. Elle représente globale- ment $5 \%$ du produit national brut. Toutefois, il est important de souligner qu'elle n'est pas très élastique contrairement à la définition de l'activité de tourisme.

En effet, les voyages sont motivés par des considérations spécifiques, trẻs souvent peu reliées aux conditions particulières d'hébergement. On pourra investir des dizaines de millions de dollars dans les centres de ski du Québec sans atténuer l'attrait du soleil des Antilles ou des montagnes du Colorado. De même, les plus belles campagnes de publicité pour attirer le tourisme d'affaires à Montréal ne changeraient pas le fait que les sièges sociaux des grandes entreprises sont localisés à Toronto et que leur présence plutôt que la publicité déterminerait lẹ lieu des réunions d'affaires.

\section{L'impact économique du tourisme}

En soi, le tourisme est une activité économique parmi d'autres et son importance refléte l'augmentation croissante des déplacements dans nos sociétés modemes. Par le fait même, ceci ne rend 
pas al'industrien touristique importante, car cette industrie n'est qu'une aggrégation partielle de diverses industries, tant de I'industrie pétroliere que des parcs d'amusement en passant par les hótels, le transport aérien, le taxi, les restaurants, les brasseries, etc.

Une question fort pertinente concerne la possibilité d'accroître l'activité économique d'une région par l'accroissement de la demande touristique dans cette région. C'est dans ce sens que l'on parle d'impact économique du tourisme. C'est aussi dans cette optique que le tourisme intéresse au plus haut point les gouvernements.

Dans une thèse de maitrise au département d'économique de l'Université de Montréal et qui portait sur la mesure de l'impact économique des activités touristiques, Aline Rover relevait, dans sa bibliographie, environ trente-cinq études sur la mesure de l'impact économique du tourisme ou d'activités touristiques de même qu'une vingtaine d'études d'impact économique d'événements touristiques québécoisist.

Une des conclusions surprenantes de son examen était que la grande majorité des études québécoises d'impact étaient erronées sur le plan méthodologique et qu'elles surestimaient le véritable impact des événements touristiques à l'étude.

Voyons donc les principes qui soustendent l'analyse de l'impact économique d'un événement touristique. (L'analyse peut être aussi généralisée à une attraction touristique permanente).

L'impact économique d'un événement est l'augmentation de l'activité économique totale dans une région suite à l'événement. Cette augmentation vient des apports d'argent de l'extérieur de la région, et non des dépenses des gens de la région. En effet, on sait qu'en movenne, les individus consacre $9 \%$ de leurs dépenses de consommation à des dépenses touristiques. c'est-ä-dire des dépenses faites dans le cadre d'un déplacement. La présence d'un événement touristique pourra les amener à réamẹnager leur budget, à faire plus de dépenses touristiques et moins de dépenses de d'autres types, ou encore à dépenser plus aujourd'hui et moins demain. Ces réaménagements budgétaires en soi ñaugmentent pas l'activité économique globale. II s'agit essentiellement de transfert de demande.

Toutefois, de l'angle d'une région a $\mathrm{A} w$, il y aura impact économique si des gens d'une région voisine $\mathrm{B}$ v diminuent leurs dépenses de consommation pour venir participer dans la région a $\mathrm{Am}$ à un évênement touristique. Dans ce cas, la région $\alpha A$ sénéficie d'un impact économique positif, et la région $« \mathrm{~B} n_{\text {r }}$ de l'inverse. De plus, si la présence d'un événement touristique amène les gens de la région aA $\mathrm{A}$ à dépenser plus dans leur région et moins dans la région $₫ \mathrm{~B} *$, il $\mathrm{y}$ aura aussi un déplacement de demande et un impact économique différencié, positif dans la région a $A w$ et négatif dans la région Bs.

Les études d'impact économique d'événements touristiques doivent done être circonscrites territorialement. L'impact économique est mesuré par le déplacement de la demande de l'extérieur du territoire vers le territoire. Ces déplacements représentent des injections nettes de demandes (et de fonds dans la région).

L'impact d'une injection de fonds par des touristes de l'extérieur aura un impact qui variera selon l'ampleur de l'effet amultiplicateurn.

L'injection initiale des touristes amènera des revenus additionnels aux travailleurs et aux hommes d'affaires de la région. Ces personnes dépensent une partie de ces revenus dans la région, créant ainsi des activites additionnelles. Ces nouvelles activites auront à leur tour leur propre effet multiplicateur, et comme des ondes sur I'eau, l'effet économique de l'injection des touristes se répercute dans l'ensemble économique.

L'effet multiplicateur n'est pas infini. En effet, lorsque le touriste achète de la gazoline pour sa voiture, l'impact économique régional n'est pas élevé car la gazoline provient de l'extérieur de la région. Une partie des dépenses touristiques sortent rapidement de la région. Ces afuitesw comme les économistes les appellent, viennent atténuer l'effet multiplicateur.

L'importance des effets multiplicateurs est évaluée à l'aide de simulation sur des tableaux intra-sectoriels ou tableaux input-output. Bien que cette méthode ait des limites et contraintes, il reste qu'il s'agit de la mesure la plus précise des multiplicateurs. Au Québec, le Bureau de la statisticue du Québec peut rapidement simuler l'impact économique de toute injection externe de fonds.

L'effet multiplicateur variera selon la nature des depenses touristiques, et celleci variera selon les caractéristiques des événements. II est toutefois beaucoup plus bas que l'on croit généralement. Ainsi le B.S.O. avait estime que pour \$1 million injectés dans l'économie québécoise par des visiteurs de l'extérieur en 1980, il en résultait une hausse de $\$ 852,000$. du PNB, soit un multiplicateur de dépenses de $0,85^{(7)}$. Pourquoi est-il ainsi? En particulier parce que $\$ 355,000$. d'importation auraient été générés par ces dépenses touristiques.

Un multiplicateur de 0,85 est typique dans le secteur touristique. Les multiplicateurs couramment utilisés de 1,5 et 2,0 m'ont aucune justification pour les dépenses touristiques.

Une autre erreur majeure de plusieurs études d'impact économique est de considérer non seulement les dépenses des touristes de l'extérieur de la région, mais aussi les dépenses des touristes de la région à l'étude dans le calcul des effets ou des impacts. Les dépenses de ces touristes alocauxs ne doivent pas être considé rés car elle ne sont déplacées que d'un poste budgétaire à l'autre. Une dernière erreur est de considérer comme injection les dépenses de la totalité des touristes externes. En fait, on peut présumer qu' un certain nombre d'entre eux viendrait sans événement. Seule une partie des dépenses doit être comptée.

En fait, les trois effets xinitiauxw qui doivent être considérés sont les suivants :

1) dépenses des touristes qui sont venus spécifiquement à cause de l'óvéne. ment;

2) dépenses des touristes associés à des séjours prolongés à cause de l'êvênement:

3) dépenses locales des gens de la région, qui en l'absence de l'événement. auraient été en voyage à l'extérieur de la région.

La région à l'étude est définie arbitrairement. Le Québec est une unité territoriale fréquemment utilisée ici. On peut toutefois utiliser le Canada. II est aussi possible d'utiliser une sous-région, telle la Gaspésie, en se rappelant deux choses. Premièrement, il $n^{2} y$ a pas d'estimés valables des multiplicateurs pour de telles régions, sauf qu'on sait qu'ils sont inférieurs ă ceux du Québec simplement parce qu'il s'agit d'une sous-région. Deuxièmement, on devrait aussi calculer l'impact négatif d'un tel événement dans les autres régions du Québec, d'où proviennent une partie des dépenses touristiques.

Les études d'impact qui utilisent une méthodologie rigoureuse indiquent usuellement des effets économiques modestes associés à des événements touristiques. II en est de meme des études sur les attractions touristiques permanentes. Ces résultats ne sont pas surprenants. Un examen rapide des régions à haute intensité touristique illustre clairement le peu d'impact moteur du tourisme sur I'économie régionale. Ainsi au Québec, la première région touristique est celle des Laurentides: il s'agit d'une région aux prises avec des problèmes économiques chroniques. La même chose siobserve dans les régions touristiques spécialisées dans les vacances soleil, tels les Caraïbes, le Mexique, I'Afrique du Nord, etc. La Floride était en stagnation jusqu'à ce que les touristes $\mathrm{s}^{\prime} \mathrm{y}$ transforment en résidents permanents. Même dans les grandes villes, le tourisme n'a pas un impact élevé: toutefois son impuissance y est masquée par le dynamisme des autres fonctions économiques des grandes villes.

Trois facteurs expliquent le faible röle 
moteur du tourisme. Premièrement, les dépenses des touristes sont relativement spécialisées : transport, hébergement, restauration, achats de bibelots, achat de billets d'entrếe à des événements, etc. Le touriste aétires son budget parcimonieusement. Deuxièmement, une partie importante des dépenses est consacrée à des biens importés de l'extérieur des régions touristiques et est donc sans grands effets. Troisièmement, les activités de travail dans le secteur touristique sont relativement à faible productivité : elles sont dans des secteurs d'emploi peu spécialisés et à faibles salaires : commerce, restauration, hờtellerie, etc.

En somme, ce n'est pas avec le tourisme qu'on construit des bconomies fortes. II $n^{\prime} y$ a pas à s'inquiéter de ce constat. D'autres facettes de notre société sont en soutien similaire. La culture n'a pas un impact économique très élevé, mais elle est importante dans une société. II en est aussi ainsi de plusieurs formes de loisir. Tout ne peut être activité économique motrice.

\section{L'intervention gouvernementale}

Toutefois nos politiciens doivent se faire élire et réélire et, consciemment ou non, ils sont constamment à la recherche de abonnes oeuvresw pour gratifier leurs électeurs. La théorie du marché politique nous enseigne que les hommes politiques tendent à satisfaire les intérêts de groupes particuliers et en faire financer le coût, en le répartissant sur Irensemble de la population à travers le processus budgétaire. Financer un évểnement touristique permet d'offrir quelque chose de tangible aux citoyens d'une région. Les contribuables de l'ensemble des régions, qui ramassent la note, ne voient pas la différence.

Q Québec 1534-1984\%, un événement majeur touristique structuré autour d'activites nautiques commémorant la adécouvertew du Canada par Jacques Cartier en 1534, illustre bien ce processus. Les gouvernements fédéral et provinciaux dépenseront environ $\$ 110$ millions pour cet événement. Oubliant pour l'instant les effets â long terme et retenant un multiplicateur généreux de 1,0 , il faudrait que l'évênement suscite à l'été 1984 5,000,000 nuitées additionnelles au Québec pour générer $\$ 110$ millions de retombées économiques. L'impact touristique anticipé est toutefois beaucoup plus faible, les nuitées additionnelles anticipées au Québec n'étant que de l'ordre de quelques centaines de milliers.

Mais nos politiciens ne sont pas en peine d'explications. Ils soulignent qu'une partie importante du $\$ 110$ millions représente des investissements à long terme, comme dans l'aménagement du Vieux-Port de Québec et l'aménagement de marinas. Cet argument sonne faux. II y aurait du vrai dans la mesure où une partie des fonds peut venir des contribuables de Toronto. Toutefois, le gouvernement fédéral, et donc les contribuables québécois, paient aussi pour les fetes du tricentenaire de Toronto, ce qui annule la contribution des contribuables torontois a nos fâtes. Mais de plus, la majeure partie du financement vient du gouvernement du Québec, et donc des contribuables québéccois. II ne fait nul doute que l'impact économique aurait été beaucoup plus grand si les gouvernements avaient laissé l'argent dans les poches des contribuables au lieu de les enfouir dans le béton de quelques marinas.

Même sous l'angle d'une meilleure répartition du revenu national entre riches et pauvres, il semblerait que le financement public des projets d'aménagement et d'événements touristiques soit régressif et qu'il serait plus équitable de laisser l'argent dans les poches des contribuables.

Ce n'est toutefois pas aujourd'hui qu'on arrêtera les politiciens de dépenser l'argent des contribuables. Malartic a son camping régional; Québec, son carnaval: Montréal, son Stade Olympique; Ottawa, son Canal Rideau et ses musées; Sorel, sa marina et Chambly, son vieux fort. En avant les jeux; les gouvernements sont généreux. Même les pauvres Romains ne voulaient pas croire que derrière les largesses de leurs empereurs bedonnants, il y avait le travail de la masse et les prévarications des personnes au pouvoir qui prétendaient être généreux.

Le financement public d'un grand nombre, si ce n'est la majorité, des aménagements touristiques et des événements touristiques ne se justifie pas économiquement. Mais le public se fait leurrer car, trop souvent, il pense que c'est l'argent des autres que le gouvernement dépense. La théorie des marchés politiques et les études empiriques sur le sujet contredisent ces vues. Les deniers publics sont distribues de façon relativement proportionnelle à la distribution de la population. Dans une démocratie, les forces s'équilibrent.

Rien ne semble toutefois pouvoir freiner I'ardeur des politiciens qui recherchent la faveur du public. C'est ainsi que cette année, à l'occasion des fêtes de la StJean et du Canada, les Montréalais ont pû goûter au dernier cri des jeux de nos empereurs modernes, l'illumination du ciel de nuit par des jets de laser, à $\$ 25,000$ l'événement. Le gouvernement municipal a fait son spectacle à Terre des Hommes, le gouvernement provincial a fait le sien au Parc Maisonneuve Idans l'est, aidéologie obligew); et le fédéral au Vieux Port, asan propriété.

Le tout a coúté peut-être $\$ 0,10$ a chaque contribuable du Montréal métropolitain. c'est relativement peu, soit cinq fois moins que l'intérêt quotidien sur les installations du Stade Olympique. Mais en fin d'année. le total peut être imposant.

II ne faudrait pas croire que la majorité des argents des contribuables vont à se taper des foires au laser ou des stades olympiques. En fait, tout comme l'industrie touristique est un amalgame disparate, les flux d'argent gouvernementaux viennent aussi de sources disparates, c'est-â-dire d'un grand nombre de ministères et d'organismes.

Ainsi, I'industrie touristique bénéficie de prêts garantis. D'autres ministères créent des emplois temporaires en aménageant des campings, des circuits pédestres ou des marinas. Certains ministères rafistolent des vestiges historiques. Le plus insignifiant des députés ả une imagination débordante lorsqu'il s'agit de dépenser de l'argent dans son comté. Les aménagements touristiques ont l'avantage d'être voyants et beaucoup plus unoblesn que les chèques d'assurance-chômage.

A l'échelle canadienne, la dépense globale se situe annuellement vraisemblablement entre $\$ 500$ millions et $\$ 1$ milliard soit de $\$ 25$ à $\$ 50$ par habitant. Chaque région peut ainsi déterminer la quote part que le marché politique devrait lui fournir annuellement en moyenne (les fluctuations annuelles régionales peuvent toutefois être importantes). Ainsi, I'AbitibiTémiscamingue, population de 150,000 habitants, devrait recevoir entre $\$ 3,5$ et $\$ 6,0$ millions en projets touristiques : Canada au travail, Québec au travail, crédit touristique, subventions spéciales de Premier Ministre, subventions spéciales de députés, octroi pour les jeux de ci ou de ça, programmes ABCD de l'OPDQ, programme $X Y Z$ du MEIR, etc. Toutefois, en Abitibi-Témiscamingue comme ailleurs, c'est l'argent des contribuables quil revient, et à l'échange, les contribuables sont perdants.

Les budgets des organismes touristiques officiels touchent toutefois peu aux aménagements et aux grands événements spéciaux. En effet, les gouvernements canalisent à travers leurs ministères. offices, ou direction du tourisme, les fonds pour les dépenses ou activités dont l'attrait politique est moindre. Ainsi, le gouvernement du Canada consacre $\$ 42$ millions à Tourisme Canada, soit $\$ 2$ per capita. Le gouvernement du Québec consacrera en $1984-85, \$ 53,4$ millions ou $\$ 8$ per capita à la Direction du tourisme du ministère de l'Industrie, du Commerce et du Tourisme. Le total est donc de $\$ 10$ per capita.

Ces budgets officiels servent à plusieurs fins. Premierement, ils servent à financer des campagnes de publicité à l'extérieur du pays. C'est environ le tiers des fonds. Deuxiemement, ils servent à subventionner de la recherche et des études de toutes sortes. C'est peut-être $10 \%$ des budgets qui sont consacrés à ces fins. Troisième- 


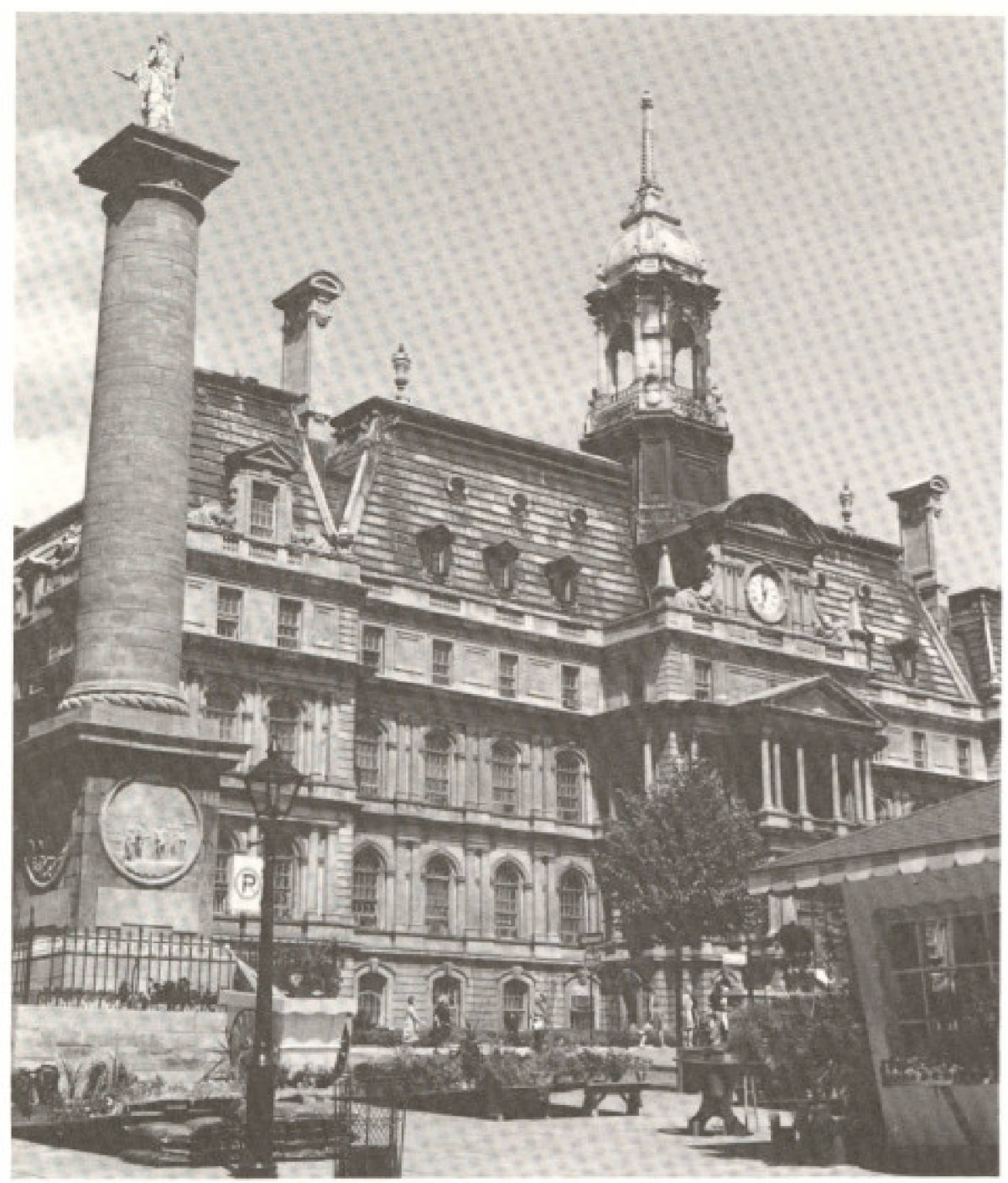

Le Vieux-Montréal a âtế négligé par nos politiciens qui prêfêrent les choses plus voyantes.

ment, ils servent à financer des organismes locaux, régionaux et nationaux de développement, tels les ATR et la Société du Palais des congrès de Montréal. Moins de $20 \%$ des budgets sont utilisés à cette fin. Enfin, ils servent à entretenir les fonctionnaires du tourisme. lls sont près de 500 à Québec et quelques centaines à Ottawa. Evidemment, ces budgets ne tiennent pas compte des parcs, qui relèvent de d'autres ministères. Seulement à Québec, plus de $\$ 100$ millions sont consacrés aux parcs et à la faune. A Ottawa, le budget des parcs nationaux est beaucoup plus important, et une grosse partie de ce budget devrait être considérée comme une dépense touristique.

\section{Conclusion}

Les intervenants de l'industrie touristique sont de grands gagnants à court terme de cette sollicitude des politiciens. En effet. avant d'atteindre le peuple, la amannew passe par leurs mains. Toutefois, la société est vraisemblablement perdante. La rentabilité sociale de ces investissements est peu élevée. Tous les investisse- ments et dépenses des gouvernements dans le secteur touristique ne sont certes pas injustifies. En fait, un grand nombre sont forts valables. Mais un nombre croissant d'interventions ne se justifie pas sur le plan économique. Le tourisme sert en quelque sorte de véhicule pour le marché politique, comme le pavage des rues principales de village ou la construction de ponts l'étaient, il y a cinquante ans.

A long terme, rindustrie touristique peut être perdante en se mêlant à ce jeu, et ce à plusieurs titres. D'une part, l'intervention gouvernementale a souvent très peu de rationalité touristique. Au nom du tourisme, Montréal a eu l'Expo 67, les Jeux Olympiques et ces équipements, le combat Léonard-Duran, les Floralies, le Vieux-Port, et pour l'an prochain, une ferme sur l'ile Notre-Dame. L'ampleur des investissements consentis ne peut masquer l'incohérence de l'ensemble des interventions. En fait, on peut dire qu'à Montréal, le gouvernement a pris en charge le développement touristique.

Le goût des politiciens pour le spectaculaire peut nuire grandement à un aménagement national de notre patrimoine touristique. Un exemple parmi tant d'autres : le Vieux-Montréal, une richesse architecturale unique en Amérique du Nord, qui ne se prête pas toutefois au spectaculaire et au tape-à-l'oeil. Le VieuxMontréal a été négligé par nos politiciens. qui préferent les choses plus voyantes. En somme, il y a un grand danger à imposer la rationalité des hommes politiques au développement touristique.

Le deuxième danger est le développement d'une dépendance face aux gouvernements et à son financement. Prenons pour exemple, le cas de la publicité. Qutarriverait-il si, pour des raisons d'Etat, les gouvernements cessaient de publiciser nos katoursy à l'étranger ou encore, s'ils empruntaient un axe de communication qui pour quelque raison, ne serait pas acceptable? Une trop grande dépendance est toujours une position stratégique vulnérable. Le financement par les gouvernements développe aussi des attitudes de dépendance. Au lieu de chercher du capital auprès des individus ou des institutions, nombreux sont les promoteurs de projets touristiques qui préfèrent faire l'antichambre pour obtenir une subvention.

L'industrie touristique est de plus en plus affectée par ce mal pernicieux. À long terme, cette dépendance peut être fatale. Les industries dynamiques sont rarement asservies à des régimes de subvention. En effet, la créativité de l'entrepreneur ne résiste pas à un climat de dépendance. A cet égard, les grandes villes touristiques, Paris, New York, Florence, Rome et San Francisco, ont su échapper à cet état.

Un milieu de vie intéressant peut bénéficier d'un apport financier des gouvernements pour sa mise en valeur. Mais il y a danger de perdre de vue l'essentiel de l'accessoire lorsque les subventions deviennent l'élément déterminant d'une politique touristique. Le touriste se retrouve alors dans un milieu de vie artificiel, concu pour luil, en carte postale. Et lentement, l'attrait touristique de la région s'atrophie. C'est le mal qui guette à long terme les régions qui se gavent de subventions. $f$

\section{Réfêrences}

(1) Office du tourisme du Canada, Le tourisme au Canada : le passê, le présent et Tavenir, Ottawa 1982.

(2) Statistique Canada, 66-201.

(3) O.C.D.E., wTourism Policy and International Tourismm, Paris 1983.

(4) SECOR, wLe defi des annees 'B0w, ACTA 1989.

(5) U.S. Data Travel Center, National Traval Survey, Washington, 1980.

(6) ROYER, Aline, L'évaluation économique d'évênement à caractêre touristique, thetses de maitrise, Université de Montréal, 1983.

(7) Bureau de la statistique du Québec - Etude d'impact économique concernant les dít penses touristiques occasionnetes par les Floralies Internationales de Montróal qui auront lieu en 1980, Octobre 1979. 